\title{
Coefficient Bound of a New Generalized Class of Tilted Analytic Univalent Functions
}

\author{
Abdullah Yahya ${ }^{1 *}$, Mohd Najir Tokachil ${ }^{1}$, Hasilatul Hana Hamzah², Shahriel Mohd Saifullah Pirman ${ }^{3}$, Siti Asiah Che Muhammad ${ }^{4}$ \\ ${ }^{1}$ Faculty of Computer and Mathematical Sciences, Universiti Teknologi MARA, Seremban, 70300, Malaysia \\ ${ }^{2}$ J.K Wire Harness Sdn. Bhd. No. 7 and 9, Kawasan Perindustrian Tebrau 1, Johor Bahru, 81100, Malaysia \\ ${ }^{3}$ Sekolah Menengah Kebangsaan Jambatan Putih, Tawau, 91019, Malaysia \\ ${ }^{4}$ Power Cables Malaysia, Purchasing Department, Shah Alam, 40702, Malaysia \\ *Corresponding author: abdullahyahya@uitm.edu.my
}

\section{Abstract}

This paper is concerned with the new generalized class of tilted analytic univalent functions, $S^{*}(\theta, \alpha, s, t)$ which denoted as.

$$
\operatorname{Re}\left\{e^{i \theta} f^{\prime}(z) \frac{z}{m(z)}\right\}>\alpha
$$

for $\cos \theta>\alpha,|\theta|<\pi, 0 \leq \alpha<1, m(z)=\frac{z}{(1-s z)(1-t z)}, s \neq 1,-1 \leq t<1, s \neq t$ and $s, t \in \mathbb{C}$ which is analytic in the unit disk $\Delta=\{\mathrm{w}:|\mathrm{w}|<1\}$. The coefficient bound as well as representation theorem of extremal properties is obtained $S^{*}(\theta, \alpha, s, t)$.

\section{Keywords}

Analytic Functions, Univalent Functions, Representation Theorem, Coefficient Bound

Received: 15 September 2021, Accepted: 02 January 2022

https://doi.org/10.26554/sti.2022.7.1.67-72

\section{INTRODUGTION}

Let $\mathbf{U}$ represent the class of functions presented by

$$
f(z)=z+a_{2} z^{2}+a_{3} z^{3}+\ldots+a_{n} z^{n}+\ldots
$$

which are regular in $\Delta=\{w:|w|<1\}$ and is the subclass of $\mathbf{U}$ consisting of normalized univalent functions which are satisfying $f(0)=0$ and $f^{\prime}(0)-1=0$ (Goodman, 1983; Duren, 2001). We also denote the subclass of $\mathbf{U}$ containing functions that are starlike, convex, and close-to-convex by $S t, K$ and $C$ respectively. Silverman and Silvia (1996) obtained representation theorem and coefficient bound for the class $G(\delta, 0)$ satisfies $\operatorname{Re}\left(e^{i \delta} \frac{f^{\prime} z}{h^{\prime} z}\right)>0|\delta|<\frac{\pi}{2}, h(z) \in K$ and $z \in \Delta$. Subsequently, Mohamad (1998) introduced the class $G(\lambda, \gamma)$ satisfies $\operatorname{Re}\left(e^{i \lambda} \frac{f^{\prime} z}{h^{\prime} z}\right)$ $>\gamma$,where $|\lambda| \leq \pi, \cos \lambda-\gamma>0$ and $z \in \Delta$ which is the same $h,(z)$ done by Silverman and Silvia (1996) and discovered representation theorem and coefficient bound of $G(\alpha, \delta)$. Afterward, Soh and Mohamad (2006) determined extremal properties for the class of functions which $h(z)=-z-2(\log (1-z))$ implies $n^{\prime}(z)=$ $(1+(z))(1-z)^{-1}$ where it is the extreme function produced by MacGregor (1962). Next, Cik Soh (2009) introduced the class $G(\lambda, \gamma, \gamma)$ with the form of $k_{\gamma}(z)=-z(1-2 \gamma)-(\log (1-z))(2-2 \gamma)$ where $0 \leq \gamma \leq 1 g^{\prime}(z)=\frac{1+(1-2 \gamma) z}{1-z}$ and the author presented some

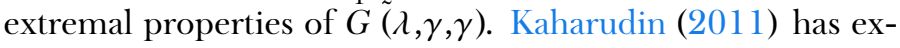

panded the results from Cik Soh (2009) by presenting a special case of $g_{\beta}(z)$ when $\beta=\frac{1}{2}$ given by Brickman et al. (1973) and found important properties of the coefficient bound and the rotation theorem for this class of functions. In addition, many researchers such as MacGregor (1962), Goel (1967), MacGregor (1964), Silverman (1972), Goel and Mehrok (1983), Silverman and Telage (1979), Fukui et al. (1987), Yahya et al. (2014), Kaplan (1952), Mohamad (2000), Akbarally et al. (2011), Wang (2010), Shashkin (1994), Çağlar et al. (2013), Magesh and Yamini (2013), Elhaddad and Darus (2020), Adegani et al. (2021), and Shaba and Wanas (2022) had studied geometrical properties of the different class of functions.

In the present paper, we introduce a new generalized class of tilted analytic univalent functions $S^{*}(\theta, \alpha, s, t)$ as the class of normalized functions $f \in \mathbf{U}$ fulfilling the condition

$$
\operatorname{Re}\left(e^{i \theta} \frac{z f^{\prime}(z)}{m(z)}\right)>\alpha,(z \in \Delta)
$$

for $\cos \theta>\alpha,|\theta|<\pi, 0 \leq \alpha<1, m(z)=\frac{z}{(1-s z)(1-t z)}, s \neq 1,-1 \leq$ $t<1, s \neq t$ and $s, t \in \mathbb{C}$. Thus, our purpose in the present paper is to obtain representation theorem and coefficient bound of $S^{*}(\theta, \alpha, s, t) \mathrm{f}$. 


\section{REPRESENTATION THEOREM}

Suppose that $P$ is the class of all functions with a positive real part $\Delta=\{\mathrm{w}:|\mathrm{w}|<1\}$. Thus, we can write the function $p(z)$ $\in P=p(z)=1+p_{1} z+p_{2} z^{2}+\ldots+p_{n} z^{n}+\ldots$ as that is analytic in $\Delta=\{w:|w|<1\}$ such that $\operatorname{Re}\{p(z)\}>0$. Next, we relate the functions $S^{*}(\theta, \alpha, s, t)$ to the functions in $P$. For any $f \in \mathbf{U}$, we have

$$
e^{i \theta} \frac{\frac{z f^{\prime}(z)}{m(z)}-\alpha-i \sin \theta}{\cos \theta-\alpha}=P,(z, \in, \Delta)
$$

The approach of The Herglotz Representation Theorem will be carried on for the establishment of the representation theorem $S^{*}(\theta, \alpha, s, t)$

\subsection{Theorem}

Let $f \in S^{*}(\theta, \alpha, s, t)$, then some probability measure $\mu$ on the unit circle $X f$ can be shown as

$$
\begin{aligned}
f(z)=\int_{x} & {\left[\left[\frac{\log (1-x s z)}{x}-\frac{\log (1-x t z)}{x}\right]+\left(2 A_{\theta \alpha} e^{-1 \theta}\right)\right.} \\
& {\left[-\frac{\log (1-x z)}{(s-1)-(t-1) x}+\log (1-x s z) \frac{s}{(t-s)-(s-1) x}-\frac{t \log (1-x t z)}{(t-s)-(t-1) x}\right] } \\
& \left.\left(\frac{2 \alpha e^{i \theta-e^{-2 i \theta}}}{(t-s)}\right)\right] d \mu(x)
\end{aligned}
$$

and conversely, if $t$ is given by the (3), then $f \in S^{*}(\theta, \alpha, s, t)$.

\section{Proof.}

We have $p \in P$ which is $p(z)=\int_{x}(1+x z)(1-x z)^{-1} d \mu(x),|x|=1$, for some probability measure $\mu$ on the unit circle $X$. Rearranging yields,

$$
e^{i \theta} \frac{z f^{\prime}(z)}{m(z)} z=p(z)(\cos \theta-\alpha)+\alpha+i \sin \theta,
$$

and by replacing $\cos \theta-\alpha=A_{\theta \alpha}$ that is always positive, we have

$$
f^{\prime}(z)=\frac{m(z)}{z e^{i \theta}}\left[A_{\theta \alpha} p(z)+\alpha+i \sin \theta\right]
$$

Therefore,

$$
f^{\prime}(z)=e^{-i \theta} \frac{m(z)}{z}\left[A_{\theta \alpha} p(z)+\alpha+i \sin \theta\right]
$$

Substituting $m(z)=\frac{z}{(1-s z)(1-t z)}$ into (4), we have

$$
f^{\prime}(z)=e^{-i \theta}\left(\frac{z}{(1-s z)(1-t z)}\right)\left(\frac{1}{z}\right)\left[A_{\theta \alpha} p(z)+\alpha+i \sin \theta\right]
$$

and

$$
f^{\prime}(z)=e^{-i \theta}\left(\frac{1}{(1-s z)(1-t z)}\right)\left[A_{\theta \alpha} p(z)+\alpha+i \sin \theta\right]
$$

From, we have

$$
f^{\prime}(z)=e^{-i \theta}\left(\frac{1}{(1-s z)(1-t z)}\right)\left[A_{\theta \alpha} \int_{x} \frac{(1+x z)}{(1-x z)} d \mu(x)+\alpha+i \sin \theta\right]
$$

Then, we obtain

$$
f^{\prime}(z)=\int_{x} e^{-i \theta}\left[\frac{(\alpha+i \sin \theta)(1-x z)+(1+x z) A_{\theta \alpha}}{(1-x s z)(1-x t z)(1-x z)}\right] d \mu(x)
$$

It follows that

$$
\begin{aligned}
f(z)= & \int_{0}^{z} \int_{x} e^{-i \theta}\left[\frac{A_{\theta \alpha}(1+x \varphi)+(\alpha+i \sin \theta)(1-x \varphi)}{(1-x s \varphi)(1-x t \varphi)(1-x \varphi)}\right] \\
& d \mu(x) d \varphi
\end{aligned}
$$

and

$$
f(z)=\int_{\substack{0 \\ d \mu(x) d \varphi}}^{z} e^{-i \theta}\left[\frac{(\cos \theta+x \varphi \cos \theta-\alpha-x \varphi \alpha+i \sin \theta-x \varphi i \sin \theta+\alpha-x \varphi \alpha)}{(1-x s \varphi)(1-x t \varphi)(1-x \varphi)}\right]
$$

Then,

$f(z)=\int_{0}^{z} \int_{x} e^{-i \theta}\left[\frac{(\cos \theta+i \sin \theta)+x \varphi(\cos \theta-i \sin \theta)-2 x \varphi+\alpha-x \varphi \alpha)}{(1-x s \varphi)(1-x t \varphi)(1-x \varphi)}\right] d \mu(x) d \varphi$

Since $\cos a+i \sin a=e^{i a}$ and $\cos a-i \sin a=e^{-i a}$

$$
f(z)=\int_{0}^{z} \int_{x}\left[\frac{1+x \varphi\left[\left(e^{-2 i \theta} \cos \theta\right)-2 \alpha\left(e^{-i \theta}\right)\right]}{(1-x s \varphi)(1-x t \varphi)(1-x \varphi)}\right] d \mu(x) d \varphi
$$

Upon simplification (6), we have

$$
f(z)=\int_{0}^{z} \int_{x}\left[\frac{\left(e^{-i \theta}-2 \alpha\right) e^{-i \theta}+1}{(1-x s \varphi)(1-x t \varphi)(1-x \varphi)} \frac{-\left(e^{-i \theta}-2 \alpha\right) e^{-i \theta}}{(1-x s \varphi)(1-x t \varphi)}\right] d \mu(x) d \varphi
$$

By changing the integration order, we obtain that

$$
\begin{aligned}
f(z)=\int_{x}\left[\int _ { 0 } ^ { z } \left[\frac{\left(-e^{-2 i \theta}+2 \alpha e^{-i \theta}\right)}{(t-s)}\left(-\frac{s}{(1-x s \varphi)}+\frac{t}{(1-x t \varphi)}\right)\right.\right. \\
+\left(2 A_{\theta \alpha} e^{-i \theta}\right)\left(\frac{1}{(s-1)(t-1)(1-x \varphi)}-\frac{s^{2}}{(t-s)(s-1)(1-x s \varphi)}\right. \\
\left.\left.\left.\quad+\frac{t^{2}}{(t-s)(t-1)(1-x t \varphi)}\right)\right] d \varphi\right] d \mu(x)
\end{aligned}
$$

Integrating (7) $\phi$ concerning, 


$$
\begin{aligned}
f(z)= & \int_{x}\left[\left[\frac{\log (1-x s z)}{(x)}-\frac{\log (1-x t z)}{(x)}\right]\right. \\
+ & \left(2 A_{\theta \alpha} e^{-i \theta}\right)\left[-\frac{\log (1-x z)}{(s-1)(t-1) x}+\frac{s \log (1-x s z)}{(-s+t)(s-1) x}\right. \\
& \left.\left.\quad-\frac{t \log (1-x t z)}{(-s+t)(t-1) x}\right]\left(\frac{2 \alpha e^{-i \theta}-e^{-2 i \theta}}{(-s+t)}\right)\right] d \mu(x)
\end{aligned}
$$

which $|x|=1$, and this is desired representation.

\subsection{Corollary}

The extreme points $S^{*}(\theta, \alpha, s, t)$ are the unit points masses

$$
\begin{aligned}
f_{x}(z)=\int_{x} & {\left[\left[\frac{\log (1-x s z)}{(x)}-\frac{\log (1-x t z)}{(x)}\right]+\left(2 A_{\theta \alpha} e^{-i \theta}\right)\right.} \\
& {\left[-\frac{\log (1-x z)}{(-1+s)(-1+t) x}+\frac{s \log (1-x s z)}{(-s+t)(-1+s) x}\right.} \\
& \left.\left.-\frac{t \log (1-x t z)}{(-s+t)(-1+t) x}\right]\left(\frac{2 \alpha e^{-i \theta}-e^{-2 i \theta}}{(-s+t)}\right)\right],
\end{aligned}
$$

with $|x|=1$. Meanwhile,

$$
\begin{aligned}
f_{x}^{\prime}(z)= & \frac{\left(-e^{-2 i \theta}+2 \alpha e^{-i \theta}\right)}{(t-s)}\left(-\frac{s}{(1-x s z)}+\frac{t}{(1-x t z)}\right) \\
+ & \left(2 A_{\theta \alpha} e^{-i \theta}\right)\left(\frac{1}{(-1+s)(-1+t)(1-x z)}\right. \\
& \left.\quad-\frac{s^{2}}{(-s+t)(-1+s)(1-x s z)}+\frac{t^{2}}{(-s+t)(-1+t)(1-x t z)}\right),
\end{aligned}
$$

is the derivatives of the extreme points for $S^{*}(\theta, \alpha, s, t)$ with $|(x)|=1$.

Thus $f$ is normalized functions since

$$
\begin{gathered}
f(0)=\int_{x}\left[\left[\frac{\log (1-x s(0))}{(x)}-\frac{\log (1-x t(0))}{(x)}\right]+\left(2 A_{\theta \alpha} e^{-i \theta}\right)\right. \\
{\left[-\frac{\log (1-x(0))}{(-1+s)(-1+t) x}+\frac{s \log (1-x s(0))}{(-s+t)(-1+s) x}\right.} \\
\left.\left.-\frac{t \log (1-x t(0))}{(-s+t)(-1+t) x}\right]\left(\frac{2 \alpha e^{-i \theta}-e^{-2 i \theta}}{(-s+t)}\right)\right]=0
\end{gathered}
$$

and

$$
\begin{aligned}
f^{\prime}(0) & =\frac{\left(-e^{-2 i \theta}+(2 \alpha) e^{-i \theta}\right)}{(-s+t)}\left(\frac{t}{(1-x t(0))}-\frac{s}{(1-x s(0))}\right) \\
+\left(2 A_{\theta \alpha} e^{-i \theta}\right)\left(\frac{1}{(s-1)(t-1)(1-x(0))}\right. & \left.-\frac{s^{2}}{(t-s)(s-1)(1-x s(0))}+\frac{t^{2}}{(t-s)(t-1)(1-x t(0))}\right)=1
\end{aligned}
$$

\section{MAIN RESULT}

Now, we proceed on finding coefficient bound of $S^{*}(\theta, \alpha, s, t) \mathrm{f}$.

\subsection{Theorem}

if $f \in S^{*}(\theta, \alpha, s, t)$, then

$$
\begin{aligned}
\left|a_{n}\right| \leqq & \frac{s . s^{n}+A_{\theta \alpha}\left(2 s^{n}-4 s . s^{n}\right)}{n(-s+t)(-s+1)}+\frac{A_{\theta} \alpha\left(2 t^{n}-4 t . t^{n}\right)-t^{n}+t . t^{n}}{n(-s+1)(-1+t)} \\
& +\frac{2 A_{\theta} \alpha}{n(-1+s)(-1+t)}
\end{aligned}
$$

for $n=2,3,4, \ldots$ and equality is attained for each $n$ when $f$ is an extreme point of $S^{*}(\theta, \alpha, s, t)$.

\section{Proof.}

From (7), we have

$$
\begin{aligned}
f(z)=\int_{0}^{z}\left[\int _ { x } \left[-\frac{\left(-e^{-2 i \theta}+e^{-i \theta}(2 \alpha)\right) s}{(t-s)(1-x s \varphi)}-\frac{\left(e^{-i \theta}\left(2 A_{\theta \alpha}\right)\right) s^{2}}{(t-s)(s-1)(1-x s \varphi)}\right.\right. \\
+\frac{\left(-e^{-i \theta}+(2 \alpha)\right) t e^{-i \theta}}{(-s+t)(1-x t \varphi)}-\frac{e^{-i \theta}\left(2 A_{\theta \alpha}\right) t^{2}}{(-s+t)(-1+t)(1-x t \varphi)} \\
\left.\left.+\frac{2 e^{-i \theta}\left(A_{\theta \alpha}\right)}{(-1+s)(-1+t)(1-x \varphi)}\right] d \mu(x)\right] d \varphi
\end{aligned}
$$

and

$$
\begin{aligned}
f(z)=\int_{0}^{z}\left[\int _ { x } \left[\left(-\frac{\left(-e^{-2 i \theta}+e^{-i \theta}(2 \alpha)\right) s}{(-s+t)}-\frac{\left(e^{-i \theta}\left(2 A_{\theta \alpha}\right) s^{2}\right.}{(-s+t)(-1+s)}\right)\right.\right. \\
\\
\frac{1}{(1-x s \varphi)}+\left(\frac{\left(e^{-i \theta}\left(-e^{1 \theta}+2 \alpha\right) t\right.}{(-s+t)(1-x t \varphi)}-\frac{e^{-i \theta}\left(2 A_{\theta \alpha}\right) t^{2}}{(-s+t)(-1+t)}\right) \\
\left.\left.\frac{1}{(1-x t \varphi)}+\left(\frac{e^{-i \theta}\left(2 A_{\theta \alpha}\right)}{(-1+s)(-1+t)}\right) \frac{1}{(1-x \varphi)}\right] d \mu(x)\right] d \varphi
\end{aligned}
$$

Next, (8) be written as

$$
\begin{array}{r}
f(z)=\int_{0}^{z}\left[\left(-\frac{\left(-e^{-2 i \theta}+2 \alpha e^{-i \theta}\right) s}{(-s+t)}-\frac{\left(2 A_{\theta \alpha} e^{-i \theta}\right) s^{2}}{(-s+t)(-1+s)}\right)\right. \\
\int_{x} \sum_{0}^{\infty}\left[(s x)^{n}\right] d \mu(x)(\varphi)^{n}+\left(\frac{\left(-e^{-2 i \theta}+2 \alpha e^{-i \theta}\right) t}{(-s+t)}\right. \\
\left.\quad-\frac{\left(2 A_{\theta \alpha} e^{-i \theta}\right) t^{2}}{(-s+t)(-1+t)}\right) \int_{x} \sum_{0}^{\infty}\left[(t x)^{n}\right] d \mu(x)(\varphi)^{n} \\
\left.+\left(\frac{\left(2 A_{\theta \alpha} e^{-i \theta}\right)}{(-1+s)(-1+t)}\right) \int_{x} \sum_{0}^{\infty}\left[x^{n} d \mu\right](x)(\varphi)^{n}\right] d \varphi
\end{array}
$$

From (9), substituting $n=0$, then 


$$
\begin{aligned}
f(z)= & -\frac{\left(-e^{-i \theta}+2 \alpha\right) e^{-i \theta}(-1+s)(-1+t) s}{(-s+t)(-1+s)(-1+t)} \\
& -\frac{e^{-i \theta}\left(2 A_{\theta \alpha}\right)(-1+t) s^{2}}{(-s+t)(-1+s)(-1+t)} \\
& +\frac{\left(-e^{-2 i \theta}+2 \alpha e^{-i \theta}\right)(-1+s)(-1+t) t}{(-s+t)(-1+s)(-1+t)} \\
& -\frac{\left(2 A_{\theta \alpha} e^{-i \theta}\right)(-1+s) t^{2}}{(-s+t)(-1+s)(-1+s)}+\frac{\left(2 A_{\theta \alpha} e^{-i \theta}\right)(-s+t)}{(-s+t)(-1+s)(-1+t)}
\end{aligned}
$$

and

$$
\begin{aligned}
f(z)= & \frac{1}{(t-s)(s-1)(t-1)}\left(e^{-2 i \theta} s^{2} t-2 \alpha s^{2} t-\left(e^{-2 i \theta}\right) s^{2}\right. \\
& -e^{-2 i \theta}(s t)+\left(2 \alpha s^{2}\right) e^{-i \theta}+(2 \alpha s t) e^{-i \theta}-2 \alpha s e^{-i \theta} \\
& \left.+s e^{-2 i \theta}\right)-\left(2 s^{2} t-2 s^{2}\right) A_{\theta \alpha} e^{-i \theta}+\left(s t^{2}\left(-e^{-2 i \theta}\right)\right. \\
& +2 \alpha e^{-i \theta} s t^{2}+e^{-2 i \theta} s t+e^{-2 i \theta} t^{2}-e^{-i \theta}(2 \alpha s t) \\
& -e^{-i \theta}\left(2 \alpha t^{2}\right)-e^{-2 i \theta} t+e^{-i \theta}(2 \alpha t)+\left(2 s t^{2}-2 t^{2}\right) \\
& \left.A_{\theta \alpha} e^{-i \theta}+(2 t-2 s) A_{\theta \alpha} e^{-i \theta}\right)
\end{aligned}
$$

Upon simplification the equation (10), we have

$$
\begin{aligned}
f(z)=\frac{1}{(t-s)(s-1)(t-1)}\left[\left(s^{2} t-s^{2}+s-s t^{2}+t^{2}-t\right) e^{-2 i \theta}\right. \\
+\left(-2 s^{2} t-2 s+2 s^{2}+2 s t^{2}-2 t^{2}+2 t\right) \alpha e^{-i \theta} \\
+\left(-2 s^{2} t-2 s+2 s^{2}+2 s t^{2}-2 t^{2}+2 t\right) \alpha e^{-i \theta} \\
\left.+\left(-2 s^{2} t+2 s^{2}+2 s t^{2}+2 t-2 t^{2}-2 s\right) A_{\theta \alpha} e^{-i \theta}\right]
\end{aligned}
$$

Substituting $A_{\theta \alpha}=\cos \theta-\alpha$ and $\cos \theta=\frac{e^{i \theta}-e^{-i \theta}}{2}$, thus

$$
\begin{aligned}
& f(z)=\frac{1}{(t-s)(s-1)(t-1)}\left[\left(s^{2} t-s^{2}+s-s t^{2}+t^{2}-t\right) e^{-2 i \theta}\right. \\
& +\left(-2 s^{2} t+2 s^{2}-2 s+2 s t^{2}-2 t^{2}+2 t\right) \alpha e^{-i \theta} \\
& +\left(-2 s^{2} t+2 s^{2}+2 s t^{2}-2 t^{2}+2 t-2 s\right) \\
& \left.\left(\frac{1+2 e^{-i \theta}-2 \alpha e^{-i \theta}}{2}\right)\right]
\end{aligned}
$$

and

$$
\begin{aligned}
f(z)= & \frac{1}{(t-s)(s-1)(t-1)}+\left[\left(-2 s^{2} t+2 s^{2}-2 s+2 s t^{2}-2 t^{2}\right.\right. \\
& \quad+2 t) \alpha e^{-i \theta}+\left(-s^{2} t+s^{2}-s+s t^{2}-t^{2}+t\right) e^{-2 i \theta} \\
& \quad+\left(2 s^{2} t-2 s^{2}+2 s-2 s t^{2}+2 t^{2}-2 t\right)+\left(-s^{2} t+s^{2}\right. \\
& \left.\left.\quad-s+s t^{2}-t^{2}+t\right)\right] \\
= & \frac{1}{(-s+t)(s-1)(t-1)}[(-s+t)(s-1)(t-1)] \\
= & 1
\end{aligned}
$$

Rewrite the equation (9) will yield to

$$
\begin{aligned}
& f(z)= \int_{0}^{w}\left[1-\left(\frac{\left(-e^{-2 i \theta}+2 \alpha e^{-i \theta}\right) s}{(t-s)}-\frac{\left(2 A_{\theta \alpha} e^{-i \theta}\right) s^{2}}{(t-s)(s-1)}\right)\right. \\
& \int_{x} \sum_{0}^{\infty}\left[(s x)^{n}\right] d \mu(x)(\varphi)^{n}+\left(\frac{\left(-e^{-2 i \theta}+2 \alpha e^{-i \theta}\right) t}{(t-s)}\right. \\
&\left.\quad-\frac{\left(2 A_{\theta \alpha} e^{-i \theta}\right) t^{2}}{(t-s)(t-1)}\right) \int_{x} \sum_{0}^{\infty}\left[(t x)^{n}\right] d \mu(x)(\varphi)^{n} \\
&\left.+\left(\frac{\left(2 A_{\theta \alpha} e^{-i \theta}\right)}{(s-1)(t-1)}\right) \int_{x} \sum_{0}^{\infty}\left[x^{n} d \mu\right](x)(\varphi)^{n}\right] d \varphi
\end{aligned}
$$

Integrating $\phi$ concerning gives us,

$$
\begin{aligned}
f(z)= & z-\left(\frac{\left(-e^{-2 i \theta}+2 \alpha e^{-i \theta}\right) s}{(t-s)}-\frac{\left(2 A_{\theta \alpha} e^{-i \theta}\right) s^{2}}{(t-s)(s-1)}\right) \\
& \int_{x} \sum_{2}^{\infty}\left[\frac{(s x)^{n-1}}{n}\right] d \mu(x) z^{n}+\left(\frac{\left(-e^{-2 i \theta}+2 \alpha e^{-i \theta}\right) t}{(t-s)}\right. \\
& \left.-\frac{\left(2 A_{\theta \alpha} e^{-i \theta}\right) t^{2}}{(t-s)(t-1)}\right) \int_{x} \sum_{2}^{\infty}\left[\frac{(t x)^{n-1}}{n}\right] d \mu(x) z^{n} \\
+ & \left(\frac{\left(2 A_{\theta \alpha} e^{-i \theta}\right)}{(s-1)(t-1)}\right) \int_{x} \sum_{2}^{\infty}\left[\frac{(x)^{n-1}}{n}\right] d \mu(x) z^{n}
\end{aligned}
$$

As a comparison above equation (1), we have

$$
\begin{aligned}
a_{n}= & -\left(\frac{e^{-i \theta}\left(-e^{-i \theta}+2 \alpha\right) s}{(-s+t) n}-\frac{e^{-i \theta}\left(2 A_{\theta \alpha}\right) s^{2}}{(-s+t)(s-1) n}\right) \\
& \int_{x} \frac{(s x)^{n}}{n s x} d \mu(x)+\left(\frac{e^{-i \theta}\left(-e^{-i \theta}+2 \alpha\right) t}{(-s+t) n}-\frac{e^{-i \theta}\left(2 A_{\theta \alpha}\right) t^{2}}{(-s+t)(t-1) n}\right) \\
& \int_{x}(t x)^{n-1} d \mu(x)+\left(\frac{e^{-i \theta}\left(2 A_{\theta \alpha}\right)}{n(s-1)(t-1)}\right) \int_{x} \frac{(x)^{n}}{n x} d \mu(x)
\end{aligned}
$$

and

$$
\begin{aligned}
\left|a_{n}\right|=\|- & \left(\frac{e^{-i \theta}\left(-e^{-i \theta}+2 \alpha\right) s}{(-s+t) n}-\frac{e^{-i \theta}\left(2 A_{\theta \alpha}\right) s^{2}}{(-s+t)(s-1) n}\right)(s)^{n-1} \\
& +\left(\frac{e^{-i \theta}\left(-e^{-i \theta}+2 \alpha\right) t}{(-s+t) n}-\frac{e^{-i \theta}\left(2 A_{\theta \alpha}\right) t^{2}}{(-s+t)(t-1) n}\right)(t)^{n-1} \\
& \left.+\left(\frac{e^{-i \theta}\left(2 A_{\theta \alpha}\right)}{n(s-1)(t-1)}\right)\right]\left[\int_{x}(x)^{n-1} d \mu(x)\right]
\end{aligned}
$$

Upon simplification $-e^{-2 i \theta}+2 \alpha e^{-i \theta}=1-2 A_{\theta \alpha} e^{-i \theta}$, we have 


$$
\begin{aligned}
\left|a_{n}\right|=\frac{1}{n} \mid & \left.\mid \int_{x}(x)^{n-1} d \mu(x)\right]\left[\left(\frac{\left(2 A_{\theta \alpha}\right) s^{2} e^{-i \theta}}{(-s+t)(s-1)}-\frac{\left(1-\left[e^{i \theta}\right] 2 A_{\theta \alpha}\right) s}{(-s+t)}\right)\right. \\
& (s)^{n-1}+\left(\frac{\left(1-2 e^{-i \theta} A_{\theta \alpha}\right) t}{(-s+t)}-\frac{e^{-i \theta}\left(2 A_{\theta} \alpha\right) t^{2}}{(-s+t)(t-1)}\right)(t)^{n-1} \\
& \left.+\left(\frac{e^{-i \theta}\left(2 A_{\theta} \alpha\right)}{(s-1)(t-1)}\right)\right] \mid
\end{aligned}
$$

and

$$
\begin{aligned}
\left|a_{n}\right|=\frac{1}{n} \mid & \mid\left[\int_{x}(x)^{n-1} d \mu(x)\right]\left[-\left(\frac{s \cdot s^{n}-4 A_{\theta \alpha} s \cdot s^{n} e^{-i \theta}-s^{n}+2 s^{n} A_{\theta \alpha} e^{-i \theta}}{(-s+t)(-1+s)}\right)+\right. \\
& \left.\left(\frac{t \cdot t^{n}-4 A_{\theta \alpha} t \cdot t^{n} e^{-i \theta}-t^{n}+2 t^{n} A_{\theta \alpha} e^{-i \theta}}{(-s+t)(-1+t)}\right)+\left(\frac{2 A_{\theta \alpha} e^{-i \theta}}{(-1+s)(-1+t)}\right)\right] \mid
\end{aligned}
$$

Then, since $\left|e^{-i \theta}\right|=1$,

$$
\begin{aligned}
& \left|a_{n}\right|=\frac{1}{n} \mid\left[\int_{x}(x)^{n-1} d \mu(x)\right]\left[-\left(\frac{s . s^{n}-s^{n}+\left(2 s^{n}-4 s . s^{n}\right) A_{\theta \alpha} e^{-i \theta}}{(-s+t)(-1+s)}\right)\right. \\
& +\left(\frac{t . t^{n}-t^{n}+\left(2 t^{n}-4 t . t^{n}\right) A_{\theta \alpha} e^{-i \theta}}{(-s+t)(-1+s)}\right) \\
& \left.+\left(\frac{2 A_{\theta \alpha} e^{-i \theta}}{(-s+t)(-1+s)}\right)\right]\left|\leq \frac{1}{n} \int_{x}\right|(x)^{n-1} \mid d \mu(x) \\
& {\left[\frac{s . s^{n}-s^{n}+\left(2 s^{n}-4 s \cdot s^{n}\right) A_{\theta \alpha}}{(-s+t)(-1+s)}+\frac{t \cdot t^{n}-t^{n}+\left(2 t^{n}-4 t \cdot t^{n}\right) A_{\theta \alpha}}{(-s+t)(-1+s)}\right.} \\
& \left.+\frac{2 A_{\theta \alpha}}{(-s+t)(-1+s)}\right] \\
& =\frac{s^{n+1}-s^{n}+\left(2 s^{n}-4 s^{n+1}\right) A_{\theta \alpha}}{(-s+t)(-1+s) n} \\
& +\frac{t^{n+1}-t^{n}+\left(2 t^{n}-4 t^{n+1}\right) A_{\theta \alpha}}{(-s+t)(-1+s) n}+\frac{2 A_{\theta \alpha}}{(-s+t)(-1+s) n}
\end{aligned}
$$

as required. Equality is attained for $n=2,3,4, \ldots$ when $f$ is an extreme point of $S^{*}(\theta, \alpha, s, t)$

\subsection{Remark}

if $f \epsilon S^{*}(\theta, \alpha, 0,0)$, we reduce to the class $G(\theta, \alpha)$ studied by Mohamad (1998) and Mohamad (2000). The coefficient bound is given by

$$
\left|a_{n}\right| \leq \frac{2 A_{\theta \alpha}}{n} \text { for } n=2,3,4, \ldots
$$

\section{CONCLUSIONS}

This paper has applied Herglotz Representation Theorem in finding representation theorem and coefficient bound for $S^{*}(\theta, \alpha, s, t)$. The results will contribute new knowledge and technique in geometric function theory.

\section{ACKNOWLEDGEMENT}

The author(s) would like to acknowledge Universiti Teknologi MARA (UiTM) Cawangan Negeri Sembilan, Kampus Seremban 3 for providing research facilities and financial support.

\section{REFERENCES}

Adegani, E. A., N. E. Cho, D. Alimohammadi, A. Motamednezhad, and I. Shahrood (2021). Coefficient Bounds for Certain Two Subclasses of Bi-Univalent Functions. AIMS Mathematics, 6(9); 9126-9137

Akbarally, A., D. Mohamad, S. C. Soh, and N. Kaharudin (2011). On The Properties of a New Class of $\alpha$-Close-to-Convex Functions. International Journal of Mathematical Analysis, 5(8); 387-396

Brickman, L., D. Hallenbeck, T. MacGregor, and D. Wilken (1973). Convex Hulls and Extreme Points of Families of Starlike and Convex Mappings. Transactions of The American Mathematical Society, $185 ; 413-428$

Çağlar, M., H. Orhan, and N. Yağmur (2013). Coefficient Bounds for New Subclasses of Bi-Univalent Functions. Filomat, 27(7); $1165-1171$

Cik Soh, S. (2009). A Generalized Class of Close-to-Convex Functions. Ph.D. Thesis, Universiti Teknologi MARA

Duren, P. L. (2001). Univalent Functions Volume 259. Springer Science \& Business Media

Elhaddad, S. and M. Darus (2020). Coefficient Estimates for a Subclass of Bi-Univalent Functions Defined by q-Derivative Operator. Mathematics, 8(3); 306

Fukui, S., S. Owa, S. Ogawa, and M. Nunokawa (1987). A Note on a Class of Analytic Functions Satisfying $\operatorname{Re}\left\{\mathrm{f}^{\prime}(\mathrm{z})\right\}>\alpha$. Bulletin of The Faculty of Education, Wakayama University. Natural Science, 36; $13-17$

Goel, R. (1967). A Class of Univalent Functions Whose Derivatives Have Positive Real Part in The Unit Disc. Nieuw Archief Voor Wiskunde, 15; 55-63

Goel, R. and B. S. Mehrok (1983). A Subclass of Univalent Functions. Journal of The Australian Mathematical Society, 35(1); 1-17

Goodman, A. (1983). Univalent Function Volume 1. Mariner Publishing Company, INC.

Kaharudin, N. (2011). On a Class of $\alpha$-Close-to-Convex Functions. Master's Thesis, Universiti Teknologi MARA

Kaplan, W. (1952). Close-to-Convex Schlicht Functions. Michigan Mathematical Journal, 1(2); 169-185

MacGregor, T. H. (1962). Functions whose Derivative has a Positive Real Part. Transactions of The American Mathematical Society, 104(3); $532-537$

MacGregor, T. H. (1964). A Class of Univalent Functions. Proceedings of The American Mathematical Society, 15(2); 311-317

Magesh, N. and J. Yamini (2013). Coefficient Bounds for Certain Subclasses of Bi-Univalent Functions. International Mathematical Forum, 8; 1337-1344

Mohamad, D. (1998). Functions Whose Derivative Maps The Unit Disc Into Half Plane. Ph.D. Thesis, University of Wales Swansea

Mohamad, D. (2000). On a Class of Functions Whose Derivatives Map The Unit Disc Into a Half Plane. Bulletin of The Malaysian Mathematical Sciences Society, 23(2); 163-167

Shaba, T. G. and A. K. Wanas (2022). Coefficient Bounds for a New Family of Bi-Univalent Functions Associated with $(U, V)$ Lucas Polynomials. International Journal of Nonlinear Analysis and Applications, 13(1); 615-626

Shashkin, Y. A. (1994). Local Degrees of Simplicial Mappings. Publicationes Mathematicae-Debrecen, 45(3-4); 407-413

Silverman, H. (1972). On a Class of Close-to-Convex Functions. Proceedings of The American Mathematical Society, 36(2); 477-484

Silverman, H. and E. Silvia (1996). On $\alpha$-Close-to-Convex Functions. 
Publicationes Mathematicae-Debrecen, 49; 305-316

Silverman, H. and D. Telage (1979). Extreme Points of Subclasses of Close-to-Convex Functions. Proceedings of The American Mathematical Society, 74(1); 59-65

Soh, S. C. and D. Mohamad (2006). On Extremal Properties for $\alpha$ Close-to-Convex Functions. Proceeding of The $2^{\text {nd }}$ IMT-GT Re- gional Conference on Mathematics, Statistics and Applications, ; 77-81 Wang, L. M. (2010). The Titled Carathodory Class and its Applications. arXiv Preprint arXiv:1003.1776

Yahya, A., S. C. Soh, and D. Mohamad (2014). Some Extremal Properties of a Generalised Close-to-Convex Function. International Journal of Mathematical Analysis, 8(39); 1931-1936 